\title{
2 DISCUSIÓN Y CONCLUSIONES
}

Tras la revisión conceptual en relación con las categorías de análisis y los instrumentos de evaluación encontrados, se identificaron 98 instrumentos en total. Sin embargo, la muestra documental obtenida se compone de instrumentos que son utilizados en el ámbito hospitalario y clínico y un porcentaje reducido de estos cuenta con algún tipo de validación para población colombiana. En el caso de algunos instrumentos recuperados, se optó por seleccionar aquellos que contaran con validación o adaptación latinoamericana como un criterio de inclusión a la muestra documental.

En su mayoría cumplen con criterios de validez y confiabilidad para población latinoamericana, pero es importante trabajar por los mismos indicadores en población colombiana para que los resultados obtenidos tengan validez respecto a la variable que evalúa. Cabe anotar que parte de este trabajo ya se había adelantado sin que se cumplieran las condiciones de confiabilidad y validez de manera estricta que se habían previsto como necesarias en el campo de la salud en general, en especial si se desea que estas sirvan como base para la evaluación de avances en procesos de intervención en salud integral (De la Fuente y Tapia, 2001).

En la misma línea de Alarcón y Muñoz (2008), los hallazgos de este estudio y las descripciones mencionadas permiten confirmar el alto grado de utilidad de los instrumentos de medición dentro del proceso de salud-enfermedad, puesto que permiten validar y complementar diagnósticos asociados con la condición psicológica y física no solo del paciente, sino del grupo de apoyo y del personal que le brinda la asistencia médica oportuna.

En el proceso de investigación y en comparación con lo encontrado con lo reportado por entidades prestadoras de salud, se observa que a pesar de recopilarse un número significativo de instrumentos utilizados en el ámbito hospitalario y clínico, en muchas 
entidades el tiempo es insuficiente para utilizar más de una estrategia de evaluación y prima la entrevista clínica. Por tanto, el empleo de los instrumentos se presenta en el desarrollo de procesos de investigación sobre problemáticas particulares.

Como ya se había anotado, es importante el contraste de información con algún instrumento de medida para establecer una línea de base objetiva y evidenciar los cambios o avances del paciente; además, se garantiza el valor de medida y la utilidad de los instrumentos (Kaplan y Saccuzzo, 2006).

En cuanto a los temas de evaluación, se identifican cuatro temáticas: autoesquemas, depresión, ansiedad y factores de riesgo, que corresponden a las áreas o los procesos identificados por Zúñiga y otros autores (1999) y son evaluados típicamente en el campo de la salud. Al analizar la relación de estas con los procesos de salud-enfermedad, se aprecia que estas son determinantes respecto al proceso de aceptación y adaptación a una condición médica.

Por otra parte, es importante resaltar e incentivar la investigación y adecuación de los instrumentos de medición psicológica en población colombiana, ya que a pesar de contar con estrategias de adaptación idiomática, algunos ítems no son aplicables debido a su contenido expreso, es decir, como lo proponen Kaplan y Saccuzzo (2006) es necesario darle mayor utilidad clínica y técnica a los instrumentos y mayor aplicabilidad a partir del empleo del lenguaje, ya sea en su uso total o en la aplicación específica de los factores que los conforman. Generalmente, los enunciados de los instrumentos aluden a situaciones particulares del país de construcción o a modismos propios, condición que implica adaptaciones -al menos en el ámbito lexicalque les ofrecerán mayor consistencia (Carvajal et al., 2011) y universalidad en su uso.

En cuanto al proceso de recuperación de información sobre los instrumentos, es importante resaltar que en su mayoría no mencionan la necesidad de que quien los aplique tenga cierta experticia para que oriente a quien lo responde, lo cual, desde la Psicometría, es un factor determinante de la validez de los resultados. Asimismo, se considera que la adaptación idiomática es determinante respecto a la utilidad del instrumento dentro del conjunto de estrategias de evaluación utilizadas por el psicólogo en los procesos de salud-enfermedad.

Acerca del manejo ético de los instrumentos, cabe señalar que aunque son muy pocos los validados en Colombia, todos los que se recuperaron tienen utilidad dentro del contexto de salud, ya que a pesar de sus características específicas en la presentación de los resultados y las dificultades idiomáticas, se convierten en un indicador inicial de la problemática del consultante. La veracidad de los resultados obtenidos de los instrumentos es parcial en el caso de aquellos que son construidos para otras 
poblaciones, pero de la mano de otras estrategias de evaluación permiten validar diagnósticos y establecer planes de intervención coherentes con las necesidades de los consultantes.

$\mathrm{Al}$ evaluar el estado de validación del reducido número de instrumentos que reportan algún análisis de este tipo - aun cuando cuentan con análisis factoriales, índices de consistencia interna altos o moderados-, es relevante que las muestras utilizadas fueron seleccionadas en grupos de conveniencia ubicados en regiones específicas del país; esto es importante si se tiene en cuenta que en todos los casos los análisis se hicieron usando constructos que corresponden a la teoría clásica de los test en los que la representatividad de las muestras es crucial. Entonces, es posible concluir que en buena parte de los casos solo puede hablarse de validaciones preliminares o selectivas.

Con el presente estudio, se hace evidente la necesidad de dedicar tiempo al desarrollo de ejercicios investigativos cuya finalidad sea caracterizar las problemáticas psicológicas propias del contexto colombiano y determinar la manera más adecuada de evaluarlas.

No obstante, los resultados ponen de manifiesto la importancia de evaluar la comorbilidad entre las condiciones médicas de carácter transitorio o crónico con las condiciones mentales de los pacientes; de allí la ventaja de poder contar con instrumentos que examinan la relación, el desarrollo o la presencia de cuadros diagnósticos mentales cuando ha surgido una condición de enfermedad física, por ejemplo, una enfermedad coronaria y su vínculo con cuadros ansiosos o enfermedades crónicas como el cáncer y la existencia de cuadros depresivos o adaptativos (Camargo y Gutiérrez, 2010; Daza, 2002; Robledo y Escobar, 2010).

El rol del psicólogo dentro del equipo interdisciplinario y multidisciplinario es fundamental en los procesos de evaluación e intervención integral, al brindar no solo un acompañamiento permanente en las fases de enfermedad, sino al contribuir al mejoramiento o mantenimiento de la calidad de vida del paciente y de su entorno. Además, incluye en la evaluación a los otros actores del sistema de salud, entre los que Schlaepfer e Infante (1990) ya habían identificado a la familia, los profesionales de la salud y al personal que apoya al cuerpo médico en la labor interventiva y son reconocidos en los resultados de esta investigación.

Los estudios centrados en la recolección y el análisis de los instrumentos psicológicos útiles en cada una de las áreas aplicadas de la Psicología (Clínica, Salud, Organizacional, Educativa, Jurídica y Comunitaria) pueden garantizar que los procesos de evaluación sean más confiables y que se puedan establecer programas de intervención acordes con los contextos correspondientes. Este tipo de investigación puede arrojar 
resultados con mayor peso investigativo, en la medida en que se retomen únicamente aquellos instrumentos cuyo reporte aparece publicado en revistas de reconocimiento científico, más que aquellas cuyo reporte se encuentra en documentos académicos (tesis y monografías, entre otros).

En Colombia, se hace necesario el desarrollo de procesos de investigación en los que se busque validar instrumentos que hayan sido aplicados y validados en diversas poblaciones, ya que esto garantiza que el instrumento cumple con criterios de validez y confiabilidad y se puede utilizar en la población. La validación por cuestiones de costos y tiempo es una alternativa viable en el caso de las investigaciones psicométricas. Para el caso particular de la Psicología de la Salud, se convierte en una buena alternativa para incrementar el número de recursos dentro del proceso de evaluación, ya que no se cuenta con un banco de instrumentos suficientes (inventarios, escalas y listas de chequeo, entre otros). 